\title{
Subsequent Effect of Lysine Regimens on Egg Characteristics of Native Aseel Chicken
}

\section{-Author(s)}

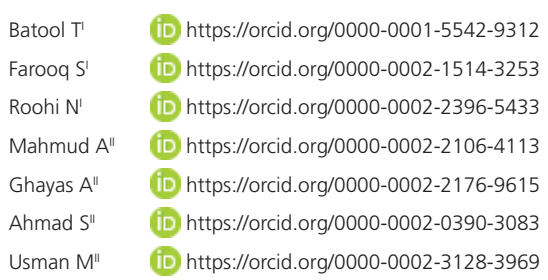

Department of Zoology, University of Punjab, Lahore, Pakistan. 54590.

" Department of Poultry Production, University of Veterinary \& Animal Sciences, Lahore, Pakistan. 54000 .

\section{Mail Address}

Corresponding author e-mail address Tahira Batool

Physiology/Endocrinology laboratory, Department of Zoology, University of the

Punjab, Lahore, 54590, Pakistan.

Phone: +92-301-4923539

Email: tahirabatool1111@gmail.com

\section{-Keywords}

Aseel, egg quality, Lysine regimens.

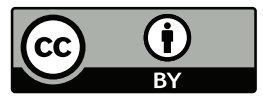

Submitted: 21/February/2018 Approved: 16/July/2018

\section{ABSTRACT}

This study was planned to evaluate subsequent effects of rearing under various lysine regimens on egg quality traits in Peshawari, Mushki, Mianwali and Lakha varieties of native Aseel. Ninety six pullets and 12 cockerels ( $24 \%$ and $\left.30^{\prime \prime}\right)$ from each variety were randomly chosen, placed in three-tiered cage units of equal space and standard conditions were followed for their maintenance. These birds were reared on three lysine regimens ( $L 1, L 2$ and $L 3)$, wherein $L 1$ containing $1.3 \%$ lysine was given in one phase from week 1-6 of age, L2 regimens entailing 1.4 and $1.2 \%$ lysine was smeared in two phases from week 1-3 and 4-6 of age, while in L3 regimen, lysine @ 1.5, 1.3 and 1.1\% in diet was fed in three phases from week 1-2, 3-4 and 5-6 of age, respectively. Data were analyzed using two-way factorial ANOVA technique in SAS 9.1 under randomized complete block design and significant means were compared through Duncan's Multiple Range Test. Statistical analysis showed that weight of egg and shell, albumen weight, diameter and index, yolk weight and Haugh units $(p \leq 0.05)$ improved significantly in L3 in Peshawari Aseel depicting the better egg quality traits. It was concluded that $\mathrm{L} 3$ regimen has positive impact on egg characteristics of Aseel chicken.

\section{INTRODUCTION}

Poultry eggs are a cheaper source to meet the nutritive requirements of the increasing human population (Hussain et al., 2013). Chicken eggs contain approximately all the essential amino acids, vitamins and minerals as well as a rich source of energy (Ahsan-ul-Haq \& Akhtar, 2002). The financial success of poultry farmer depends upon the number of eggs sold, while preference and acceptability of consumer highly depends upon egg quality parameters including egg weight, size, shell thickness, albumen height, yolk consistency and Haugh unit scores (Tumova \& Gous, 2012). The egg quality characteristics are of prime importance in poultry breeding businesses (Bain, 2005) and egg quality has several aspects based on heredities, age and strains or breeds variations (Silversides et al., 2006; Onagbesan et al., 2007). The spoilage of egg is caused by deterioration of internal egg contents and their quality (Kul \& Seeker, 2004), which is mainly instigated by poor and improper storage conditions (Adeogun \& Amole, 2004). However, the physical configuration and chemical composition of egg reveals its stability and plays a key role during storage (Seidler, 2003). The egg size, albumen and yolk deposition, internal egg contents and quality are influenced by nutritional aspects including the levels of essential amino acids. Lysine is an important amino acid being supplemented in poultry feed and has great impact on body protein as well as egg deposition (Valerio et al., 2003). In Pakistan the eggs are mainly obtained either through commercial layer or backyard indigenous poultry farms. Among backyard chicken, Aseel is the most famous breed due to the possession of strong physical structure, stamina and resistance against 
Batool T, Farooq S, Roohi N, Mahmud A, Ghayas A, Ahmad S, Usman M
Subsequent Effect of Lysine Regimens on Egg Characteristics of Native Aseel Chicken diseases and is equally adjustable in all environments (Batool, 2017). Breast meat accretion in broilers has a positive role and has been well established in past studies (Sibbald \& Wolynetz, 1986; Holsheimer \& Ruesink, 1993; Kidd et al., 1998). Later on, Leeson \& Zubair (1997) showed a decline in growth rate when feeding extra dietary lysine after feeding a deficient diet from 6 to 12 days of age. A well-defined level of dietary lysine seems necessary to be identified.

Despite of great genetic potential, Aseel is mostly kept for rooster fighting since long and its rearing, proliferation and commercialization is not preferred due to poor growth at early ages, egg production, distorted clutches and broodiness, yet it could be more economical and profitable with respect to quality egg production by adopting better house management, feed and feeding strategies (Usman et al., 2014). Keeping in view the eminence of lysine and the prominence of Aseel, the current research project was, therefore designed to improve egg quality features by applying lysine regimens during early ages in the varieties of indigenous Aseel, namely Lakha, Mianwali, Mushki and Peshawari.

\section{MATERIALS AND METHODS}

This experiment was conducted at the Indigenous Chicken Genetic Resource Center (ICGRC), Ravi campus, University of Veterinary and Animal Sciences, Lahore to estimate the subsequent impacts of rearing with three different lysine feeding regimens on egg quality characteristics among four varieties of indigenous Aseel chicken, namely Lakha, Mianwali, Mushki and Peshawari. Initially 1 day-old chicks $(n=240)$, including 60 birds of each variety were selected at random, tagged and subdivided into three groups $A, B$ and $C$ and offered three various lysine regimens i.e., L1 (1.3\% lysine), L2 (1.4, 1.2\% lysine) and L3 (1.5, 1.3, 1.1\% lysine), respectively. $\mathrm{L} 1$ constituting $1.3 \%$ lysine was offered in one phase from week 1-6, 1.4 and 1.2\% lysine in L2 was offered in two phases from week 1-3 and $4-6$, while $1.5,1.3$ and $1.1 \%$ lysine in $L 3$ was offered in three phases from week 1-2, 3-4 and 5-6, respectively. Lysine regimens and their composition are mentioned in Table 1 ( $A$ and $B$ ) and the feed was prepared from Hi-Tech Industries (Pvt) Pakistan. The study was based on the hypothesis that phasevise supplementation of lysine and other nutrients as per growth needs of birds will enhance initial growth performance which might sustain the improved egg production and quality traits as subsequent impact.
Table 1A - Chemical composition of experimental diets.

\begin{tabular}{lccccc}
\hline \multirow{2}{*}{ Ingredients } & \multicolumn{5}{c}{ Dietary lysine levels (\%) } \\
\cline { 2 - 6 } & 1.1 & 1.2 & 1.3 & 1.4 & 1.5 \\
\hline Corn & 59.08 & 59.08 & 59.08 & 59.08 & 59.08 \\
Sunflower Meal (24\%) & 18.9 & 18.9 & 18.9 & 18.9 & 18.9 \\
Soya bean Meal (44\%) & 7.04 & 7.04 & 7.04 & 7.04 & 7.04 \\
Rapeseed Meal & 3.00 & 3.00 & 3.00 & 3.00 & 3.00 \\
Fish Meal (52\%) & 3.00 & 3.00 & 3.00 & 3.00 & 3.00 \\
Poultry by-product Meal & 3.00 & 3.00 & 3.00 & 3.00 & 3.00 \\
Molasses & 3.00 & 3.00 & 3.00 & 3.00 & 3.00 \\
Limestone & 1.14 & 1.14 & 1.14 & 1.14 & 1.14 \\
Lysine Sulphate & 0.7 & 0.9 & 1.1 & 1.3 & 1.5 \\
Mono Calcium Phosphate & 0.45 & 0.45 & 0.45 & 0.45 & 0.45 \\
Vitamin-Mineral Premix* & 0.2 & 0.2 & 0.2 & 0.2 & 0.2 \\
Sodium Chloride & 0.18 & 0.18 & 0.18 & 0.18 & 0.18 \\
Alimet (Novus) & 0.17 & 0.17 & 0.17 & 0.17 & 0.17 \\
Betaine HCl & 0.05 & 0.05 & 0.05 & 0.05 & 0.05 \\
Threonine & 0.04 & 0.04 & 0.04 & 0.04 & 0.04 \\
\hline
\end{tabular}

*Vit-Min premix supplied per 1 kg of diet: Vit. A 12000 IU; Vit. D3 2200 ICU; Vit. E 10 mg; Vit. K 32 mg; Vit. B1 1 mg; Vit. B2 4 mg; Vit. B6 1.5 mg; Vit. B12 $10 \mu g$; nicotinic acid $20 \mathrm{mg}$; folic acid $1 \mathrm{mg}$; pantothenic acid $10 \mathrm{mg}$; biotin $50 \mu$ g; choline chloride $500 \mathrm{mg}$; copper 10 iron $30 \mathrm{mg}$; manganese $55 \mathrm{mg}$; zinc $50 \mathrm{mg}$; iodine $1 \mathrm{mg}$; selenium $0.1 \mathrm{mg}$.

These Aseel birds after brooding under various lysine regimens were equally offered normal broiler-grower (7-16 weeks), layer-developer (17-20), pre-breeder/ layer (21-24 weeks) and breeder/layer (25-43 weeks) feed with 20.02, 15.46, 16.50, 17.50\% and 3020, 2913, 2850, 2750kcal/kg CP and ME, respectively, formulated and prepared as per standards of NRC (1994) and recommendations as provided by Leeson \& Summers (2005). For estimation of egg quality traits, 96 Aseel birds, including 24 pullets and 12 cockerels including 3 of each variety were randomly chosen and placed in three tiered equally spaced cage units, equipped with easily removable and slanted trays for collecting fecal wastes and eggs, respectively. The factorial arrangement according to randomized complete block design (RCBD) was applied to 4 (varieties) $\times 3$ (treatments/lysine regimens) $\times 8$ [replicate/ pullet ( 1 bird in each replicate)], where in each bird was given a status of an individual experimental unit and 1 cockerel was available for mating with 8 pullets of respective variety under coops. During the whole egg laying period, limited feed and duration for feeding was practiced and most priority was given to the care and welfare of experimental birds by following the instructions as provided by the Institutional Animal Ethic Committee. The egg quality was defined as the an egg that showed acceptability toward the consumer. Seventy two eggs (fresh collection) including 18 from each variety, comprising 6 eggs/treatment group/4 weeks ( $72 \times 4=288$ eggs in total/4 phases) were selected 
Batool T, Farooq S, Roohi N,

Mahmud A, Ghayas A, Ahmad S, Usman M
Subsequent Effect of Lysine Regimens on Egg

Characteristics of Native Aseel Chicken

Table 1B - Calculated nutritional composition of experimental diets.

\begin{tabular}{|c|c|c|c|c|c|}
\hline \multirow{2}{*}{ Nutrients (\%) } & \multicolumn{5}{|c|}{ Dietary lysine level \% } \\
\hline & 1.1 & 1.2 & 1.3 & 1.4 & 1.5 \\
\hline Metabolize Energy (k calories/kg) & 2746.99 & 2753.69 & 2760.39 & 2767.09 & 2773.79 \\
\hline Dry Matter & 87.17 & 87.36 & 87.56 & 87.76 & 87.96 \\
\hline Crude Protein & 17.06 & 17.18 & 17.29 & 17.40 & 17.51 \\
\hline Crude Fiber & 6.93 & 6.93 & 6.93 & 6.93 & 6.93 \\
\hline Ash & 4.09 & 4.09 & 4.09 & 4.09 & 4.09 \\
\hline Either Extract & 3.59 & 3.59 & 3.59 & 3.59 & 3.59 \\
\hline Calcium & 0.84 & 0.84 & 0.84 & 0.84 & 0.84 \\
\hline Chloride & 0.22 & 0.22 & 0.22 & 0.22 & 0.22 \\
\hline Sodium & 0.16 & 0.16 & 0.16 & 0.16 & 0.16 \\
\hline Total phosphorus & 0.68 & 0.68 & 0.68 & 0.68 & 0.68 \\
\hline Potassium & 0.71 & 0.71 & 0.71 & 0.71 & 0.71 \\
\hline Digestible phosphorus & 0.36 & 0.36 & 0.36 & 0.36 & 0.36 \\
\hline Linoleic Acid & 1.42 & 1.42 & 1.42 & 1.42 & 1.42 \\
\hline Lysine & 1.1 & 1.2 & 1.3 & 1.4 & 1.5 \\
\hline Methionine & 0.45 & 0.45 & 0.45 & 0.45 & 0.45 \\
\hline Methionine+Cystine & 0.78 & 0.78 & 0.78 & 0.78 & 0.78 \\
\hline Digestible Arginine & 0.98 & 0.98 & 0.98 & 0.98 & 0.98 \\
\hline Digestible Tryptophan & 0.14 & 0.14 & 0.14 & 0.14 & 0.14 \\
\hline Digestible Threonine & 0.57 & 0.57 & 0.57 & 0.57 & 0.57 \\
\hline Digestible Lysine & 0.99 & 1.09 & 1.20 & 1.31 & 1.41 \\
\hline Digestible methionine & 0.42 & 0.42 & 0.42 & 0.42 & 0.42 \\
\hline Digestible Methionine + Cystine & 0.67 & 0.67 & 0.67 & 0.67 & 0.67 \\
\hline Threonine & 0.67 & 0.67 & 0.67 & 0.67 & 0.67 \\
\hline Tryptophan & 0.19 & 0.19 & 0.19 & 0.19 & 0.19 \\
\hline Arginine & 1.10 & 1.10 & 1.10 & 1.10 & 1.10 \\
\hline Cystine & 0.32 & 0.32 & 0.32 & 0.32 & 0.32 \\
\hline Digestible Cystine & 0.26 & 0.26 & 0.26 & 0.26 & 0.26 \\
\hline Valine & 0.82 & 0.82 & 0.82 & 0.82 & 0.82 \\
\hline Digestible Valine & 0.71 & 0.71 & 0.71 & 0.71 & 0.71 \\
\hline Histidine & 0.43 & 0.43 & 0.43 & 0.43 & 0.43 \\
\hline Digestible Histidine & 0.37 & 0.37 & 0.37 & 0.37 & 0.37 \\
\hline Phenylalanine & 0.78 & 0.78 & 0.78 & 0.78 & 0.78 \\
\hline Digestible Phenylalanine & 0.67 & 0.67 & 0.67 & 0.67 & 0.67 \\
\hline Leucine & 1.44 & 1.44 & 1.44 & 1.44 & 1.44 \\
\hline Digestible Leucine & 1.21 & 1.21 & 1.21 & 1.21 & 1.21 \\
\hline Isoleucine & 0.66 & 0.66 & 0.66 & 0.66 & 0.66 \\
\hline Digestible Isoleucine & 0.58 & 0.58 & 0.58 & 0.58 & 0.58 \\
\hline
\end{tabular}

and used for egg quality traits analysis. Egg weight was measured by using an electrical weighing balance capable of measuring up to $0.1 \mathrm{~g}$ and shell thickness was recorded by using micrometer screw gauge. Digital Vernier caliper was used to measure yolk height and width, and single observation was based on average of three measurements. The $\mathrm{pH}$ of albumen and yolk of the eggs was determined by digital $\mathrm{pH}$ meter, while albumen and yolk indices and Haugh unit scores were calculated by using the following formulae:

Albumen index $(\%)=$ (albumen height/ albumen width) $\times 100$

Yolk index $(\%)=($ yolk height/yolk width $) \times 100$

Haugh Unit=100[@ $\left.\log \left\{(H+7.57)-(1.7)\left(W^{0.37}\right)\right\}\right]$
Where;

$\mathrm{H}=$ Observed height of the albumen in $\mathrm{mm}$

$\mathrm{W}=$ Weight of egg $(\mathrm{g})$

The collected data was analyzed by SAS (Statistical Analysis System, version 9.1) software through factorial ANOVA (Analysis of Variance) technique (Steel et al., 1997) and results were mentioned as means and their standard errors. The comparison of treatment means was done through Duncan's Multiple Range test (Duncan, 1955) and results were considered as significant at $p \leq 0.05$. Following statistical model was employed:

$Y_{i j k}=\mu+B_{i}+T_{j}+B_{i} \times T_{j}+\varepsilon_{i j k}$

Where, 
Batool T, Farooq S, Roohi N, Mahmud A, Ghayas A, Ahmad S, Usman M Subsequent Effect of Lysine Regimens on Egg
Characteristics of Native Aseel Chicken
$Y_{i j k}=$ Dependent Variable

$\mu=$ Population Mean

$B_{i}=$ Effect of $i^{\text {th }}$ Block ( $i=1,2,3,4$; Lakha, Mushki, Mianwali, Peshawari)

$T_{j}=$ Effect of $j^{\text {th }}$ Treatment $(j=1,2,3$; dietary lysine regimens $L 1, L 2$, L3)

$B_{i} \times T_{i}=$ Interaction Effect

$\varepsilon_{i \mathrm{ik}}=$ Residual effect associated with $\mathrm{k}^{\text {th }}$ observation of $j^{\text {th }}$ treatment in $i^{\text {th }}$ block NID $\sim 0, \sigma^{2}$

\section{RESULTS AND DISCUSSION}

The impact of lysine regimens, Aseel varieties and the interactions of both on various egg quality traits have been presented in Table $2-4$. Significant $(p \leq 0.05)$ variations were found in egg weight and length both in lysine regimens and Aseel varieties, and interactions among them. The birds reared in L3 and L2 showed egg weight and length $45.58 \pm 0.37,45.35 \pm 0.34 \mathrm{~g}$ and $51.00 \pm 0.21,50.97 \pm 0.28 \mathrm{~mm}$, respectively compared to L1 lysine regimen. Among Aseel varieties, Peshawari led in egg weight and length than other varieties. These differences can be attributed to yolk protein contents most of which is considered to consist of lysine (Murphy, 1994). Like our study, phase feeding regimen for lysine amino acid was employed during phase I (week 20-43) and phase II (week 44-63) of Dekalb Delta hens by Novak et al. (2004), wherein, significantly $(p \leq 0.02)$ increased egg weights (59.02 to $60.21 \mathrm{~g}$ ) were observed during phase II when lysine intake was increased. Our findings of egg weights are in close agreement with the results of Batool et al. (2017), where three lysine phase feeding regimensL 1 (1.3), L2 (1.4,1.2), L3 $(1.5,1.3,1.1 \%$ lysine) were implemented to Aseel varieties during rearing phase and significantly $(p \leq 0.05)$ higher average egg weight was subsequently observed during production in Aseel birds reared under three phased lysine regimen i.e., L3.

Regarding egg shell quality parameters shell weight $(5.86 \pm 0.10 \mathrm{~g})$ and thickness $(0.34 \pm 0.01 \mathrm{~mm})$ was also significantly greater in birds of $L 3$ lysine regimen and Peshawari variety than those of other regimens and varieties, respectively. Differences in egg weight values in L1, L2 and L3 might be due to differences in ovulation and oviposition time (Gilbert, 1969; Etches, 1990; Alzenbarakji, 2011). Novak et al. (2004), in their study on Dekalb Delta laying hens also found that the percentage weight of wet shell was not

Table 2 - Impact of various lysine regimens, Aseel varieties and their interactions on egg quality traits in native Aseel pullets.

\begin{tabular}{|c|c|c|c|c|c|c|c|c|}
\hline \multicolumn{2}{|l|}{ Variables } & $\begin{array}{c}\text { Egg weight } \\
(\mathrm{g})\end{array}$ & $\begin{array}{l}\text { Egg length } \\
(\mathrm{mm})\end{array}$ & $\begin{array}{l}\text { Egg width } \\
(\mathrm{mm})\end{array}$ & $\begin{array}{l}\text { Shell weight } \\
\text { (g) }\end{array}$ & $\begin{array}{c}\text { Shell Thickness } \\
(\mathrm{mm})\end{array}$ & $\begin{array}{c}\text { Albumen weight } \\
\text { (g) }\end{array}$ & $\begin{array}{c}\text { Albumen height } \\
(\mathrm{mm})\end{array}$ \\
\hline \multicolumn{9}{|c|}{ Lysine Levels (\%)/Regimens (LR) } \\
\hline \multicolumn{2}{|c|}{$1.3(\mathrm{~L} 1)$} & $44.36 \pm 0.25^{b}$ & $50.31 \pm 0.21^{b}$ & $38.98 \pm 0.27$ & $5.62 \pm 0.08$ & $0.31 \pm 0.00^{b}$ & $24.21 \pm 0.17^{b}$ & $6.52 \pm 0.12$ \\
\hline \multicolumn{2}{|l|}{$1.4-1.2(\mathrm{~L} 2)$} & $45.35 \pm 0.34^{a}$ & $50.97 \pm 0.28^{a}$ & $39.09 \pm 0.29$ & $5.70 \pm 0.12$ & $0.33 \pm 0.00^{a}$ & $24.91 \pm 0.28^{b}$ & $7.14 \pm 0.21$ \\
\hline \multicolumn{2}{|c|}{$1.5-1.3-1.1(\mathrm{~L} 3)$} & $45.58 \pm 0.37^{a}$ & $51.00 \pm 0.21^{\mathrm{a}}$ & $39.28 \pm 0.18$ & $5.86 \pm 0.10$ & $0.34 \pm 0.01^{\mathrm{a}}$ & $25.03 \pm 0.37^{a}$ & $7.53 \pm 0.12$ \\
\hline \multicolumn{9}{|c|}{ Aseel Varieties (AV) } \\
\hline \multicolumn{2}{|c|}{ Lakha } & $44.67 \pm 0.26^{b}$ & $50.51 \pm 0.18^{b}$ & $38.98 \pm 0.15$ & $5.52 \pm 0.06^{b}$ & $0.32 \pm 0.01^{b}$ & $24.31 \pm 0.21^{b}$ & $6.76 \pm 0.18$ \\
\hline \multicolumn{2}{|l|}{ Mianwali } & $45.25 \pm 0.41^{\mathrm{ab}}$ & $50.79 \pm 0.35^{\mathrm{ab}}$ & $38.91 \pm 0.27$ & $5.82 \pm 0.13^{a}$ & $0.32 \pm 0.01^{b}$ & $25.16 \pm 0.37^{a}$ & $6.97 \pm 0.18$ \\
\hline \multicolumn{2}{|l|}{ Mushki } & $44.41 \pm 0.29^{b}$ & $50.29 \pm 0.25^{b}$ & $39.23 \pm 0.46$ & $5.55 \pm 0.10^{b}$ & $0.32 \pm 0.01^{b}$ & $24.05 \pm 0.25^{b}$ & $7.00 \pm 0.25$ \\
\hline \multicolumn{2}{|l|}{ Peshawari } & $46.06 \pm 0.45^{\mathrm{a}}$ & $51.46 \pm 0.18^{a}$ & $39.34 \pm 0.19$ & $6.03 \pm 0.08^{a}$ & $0.34 \pm 0.01^{a}$ & $25.34 \pm 0.36^{a}$ & $7.53 \pm 0.23$ \\
\hline \multicolumn{9}{|c|}{ Lysine Levels $(\%) /$ Regimens $\times$ Aseel Varieties $(\mathrm{LR} \times \mathrm{AV})$} \\
\hline \multirow{4}{*}{$\begin{array}{l}1.3 \\
(\mathrm{~L} 1)\end{array}$} & Lakha & $43.98 \pm 0.37 c$ & $50.59 \pm 0.25^{b c d}$ & $38.90 \pm 0.27$ & $5.38 \pm 0.07$ & $0.30 \pm 0.00^{e}$ & $24.23 \pm 0.47^{\text {cde }}$ & $6.30 \pm 0.17$ \\
\hline & Mianwali & $43.90 \pm 0.15^{c}$ & $49.58 \pm 0.15^{d}$ & $38.31 \pm 0.07$ & $5.59 \pm 0.09 b c$ & $0.33 \pm 0.01^{\mathrm{bcd}}$ & $24.02 \pm 0.13^{\text {de }}$ & $6.63 \pm 0.15$ \\
\hline & Mushki & $44.96 \pm 0.68^{c}$ & $49.94 \pm 0.35^{d}$ & $39.81 \pm 0.92$ & $5.64 \pm 0.10^{b c}$ & $0.33 \pm 0.01^{\text {bcde }}$ & $24.27 \pm 0.11^{\text {cde }}$ & $7.35 \pm 0.23$ \\
\hline & Peshawari & $44.62 \pm 0.62^{c}$ & $51.16 \pm 0.22^{\mathrm{abc}}$ & $38.87 \pm 0.32$ & $5.87 \pm 0.21^{a b c}$ & $0.31 \pm 0.01^{\mathrm{de}}$ & $24.32 \pm 0.58^{\text {cde }}$ & $6.43 \pm 0.25$ \\
\hline \multirow{4}{*}{$\begin{array}{l}1.4-1.2 \\
(\mathrm{~L} 2)\end{array}$} & Lakha & $45.06 \pm 0.28^{b c}$ & $50.24 \pm 0.33^{c d}$ & $38.92 \pm 0.34$ & $5.48 \pm 0.07 c$ & $0.34 \pm 0.01^{b}$ & $24.25 \pm 0.38^{\text {cde }}$ & $7.02 \pm 0.23$ \\
\hline & Mianwali & $45.41 \pm 0.48^{a b c}$ & $51.21 \pm 0.33^{\mathrm{abc}}$ & $38.91 \pm 0.70$ & $5.84 \pm 0.32^{\mathrm{abc}}$ & $0.31 \pm 0.00^{\text {de }}$ & $25.87 \pm 0.40^{a b}$ & $7.45 \pm 0.12$ \\
\hline & Mushki & $44.20 \pm 0.48^{c}$ & $50.48 \pm 0.72^{\mathrm{bcd}}$ & $39.27 \pm 1.05$ & $5.46 \pm 0.25^{c}$ & $0.31 \pm 0.01^{\mathrm{de}}$ & $24.02 \pm 0.27^{\mathrm{de}}$ & $6.55 \pm 0.30$ \\
\hline & Peshawari & $46.75 \pm 0.59^{a}$ & $51.96 \pm 0.23^{a}$ & $39.24 \pm 0.22$ & $6.04 \pm 0.08^{a b}$ & $0.32 \pm 0.01^{\text {bcde }}$ & $25.51 \pm 0.35^{\mathrm{abcd}}$ & $7.23 \pm 0.71$ \\
\hline \multirow{4}{*}{$\begin{array}{l}1.5-1.3-1.1 \\
\text { (L3) }\end{array}$} & Lakha & $44.98 \pm 0.50^{\mathrm{bc}}$ & $50.70 \pm 0.41^{\mathrm{bcd}}$ & $39.11 \pm 0.25$ & $5.69 \pm 0.08^{\mathrm{abc}}$ & $0.34 \pm 0.01^{b c}$ & $24.47 \pm 0.39$ cde & $7.22 \pm 0.13$ \\
\hline & Mianwali & $46.44 \pm 0.37^{a b}$ & $51.58 \pm 0.47^{a b}$ & $39.51 \pm 0.14$ & $6.03 \pm 0.20^{a b}$ & $0.32 \pm 0.00^{\text {bcde }}$ & $25.59 \pm 0.67^{\mathrm{abc}}$ & $6.82 \pm 0.29$ \\
\hline & Mushki & $44.09 \pm 0.35^{c}$ & $50.45 \pm 0.19^{\mathrm{bcd}}$ & $38.60 \pm 0.40$ & $5.55 \pm 0.16^{b c}$ & $0.34 \pm 0.01^{b}$ & $23.86 \pm 0.79 e$ & $7.69 \pm 0.32$ \\
\hline & Peshawari & $46.81 \pm 0.37^{a}$ & $51.26 \pm 0.32^{\mathrm{abc}}$ & $39.90 \pm 0.16$ & $6.18 \pm 0.06^{a}$ & $0.36 \pm 0.02^{a}$ & $26.20 \pm 0.40^{a}$ & $8.08 \pm 0.18$ \\
\hline \multicolumn{2}{|c|}{ Source of Variation } & \multicolumn{7}{|c|}{$P$-value } \\
\hline \multicolumn{2}{|c|}{ LR } & 0.0023 & 0.0212 & 0.6908 & 0.1223 & 0.0003 & 0.0367 & 0.3512 \\
\hline \multicolumn{2}{|l|}{ AV } & 0.0010 & 0.0037 & 0.6955 & 0.0020 & 0.0039 & 0.0041 & 0.3005 \\
\hline \multicolumn{2}{|l|}{$\mathrm{LR} \times \mathrm{AV}$} & 0.0159 & 0.0040 & 0.3389 & 0.7413 & 0.0877 & 0.0071 & 0.8715 \\
\hline
\end{tabular}

Values and standard units of all parameters have been mentioned as $M \pm S E$, superscripted alphabets represent significant $(p \leq 0.05)$ variations, wherein a>b>c. . 
Table 3 - Impact of various lysine regimens, Aseel varieties and their interactions on egg quality traits in native Aseel pullets.

\begin{tabular}{|c|c|c|c|c|c|c|c|c|}
\hline \multicolumn{2}{|l|}{ Variables } & $\begin{array}{l}\text { Albumen width } \\
(\mathrm{mm})\end{array}$ & Albumen $\mathrm{pH}$ & $\begin{array}{l}\text { Albumen } \\
\text { index (\%) }\end{array}$ & $\begin{array}{l}\text { Yolk weight } \\
\text { (g) }\end{array}$ & $\begin{array}{l}\text { Yolk height } \\
(\mathrm{mm})\end{array}$ & Yolk color & Yolk pH \\
\hline \multicolumn{9}{|c|}{ Lysine Levels (\%)/Regimens (LR) } \\
\hline \multicolumn{2}{|c|}{$1.3(\mathrm{~L} 1)$} & $52.69 \pm 0.26$ & $7.65 \pm 0.02$ & $13.30 \pm 0.29$ & $14.19 \pm 0.16$ & $16.65 \pm 0.22^{b}$ & $5.38 \pm 0.11$ & $6.47 \pm 0.03$ \\
\hline \multicolumn{2}{|l|}{ 1.4-1.2 (L2) } & $52.79 \pm 0.37$ & $7.61 \pm 0.03$ & $13.53 \pm 0.37$ & $14.46 \pm 0.27$ & $16.98 \pm 0.21^{\mathrm{ab}}$ & $5.52 \pm 0.09$ & $6.43 \pm 0.02$ \\
\hline \multicolumn{2}{|c|}{ 1.5-1.3-1.1 (L3) } & $53.09 \pm 0.41$ & $7.66 \pm 0.04$ & $14.19 \pm 0.15$ & $14.41 \pm 0.14$ & $17.22 \pm 0.15^{\mathrm{a}}$ & $5.44 \pm 0.15$ & $6.46 \pm 0.03$ \\
\hline \multicolumn{9}{|c|}{ Aseel Varieties (AV) } \\
\hline \multicolumn{2}{|c|}{ Lakha } & $52.54 \pm 0.24^{b}$ & $7.68 \pm 0.03$ & $13.25 \pm 0.30$ & $14.61 \pm 0.18$ & $16.55 \pm 0.17^{b}$ & $5.39 \pm 0.17$ & $6.45 \pm 0.02$ \\
\hline \multicolumn{2}{|l|}{ Mianwali } & $52.47 \pm 0.32^{b}$ & $7.66 \pm 0.04$ & $13.49 \pm 0.23$ & $14.00 \pm 0.26$ & $16.91 \pm 0.20^{b}$ & $5.56 \pm 0.13$ & $6.39 \pm 0.03$ \\
\hline \multicolumn{2}{|l|}{ Mushki } & $52.08 \pm 0.28^{b}$ & $7.62 \pm 0.04$ & $13.84 \pm 0.42$ & $14.45 \pm 0.21$ & $16.65 \pm 0.25^{b}$ & $5.33 \pm 0.17$ & $6.49 \pm 0.02$ \\
\hline \multicolumn{2}{|l|}{ Peshawari } & $54.33 \pm 0.30^{\mathrm{a}}$ & $7.58 \pm 0.03$ & $14.10 \pm 0.39$ & $14.35 \pm 0.23$ & $17.68 \pm 0.12^{\mathrm{a}}$ & $5.50 \pm 0.06$ & $6.48 \pm 0.03$ \\
\hline \multicolumn{9}{|c|}{ Lysine Levels (\%)/Regimens $\times$ Aseel Varieties $(\mathrm{LR} \times \mathrm{AV})$} \\
\hline \multirow{4}{*}{$\begin{array}{l}1.3 \\
(\mathrm{~L} 1)\end{array}$} & Lakha & $52.91 \pm 0.14^{\mathrm{cd}}$ & $7.66 \pm 0.05$ & $13.35 \pm 0.46$ & $14.13 \pm 0.23$ & $16.28 \pm 0.14^{\text {de }}$ & $5.08 \pm 0.17$ & $6.46 \pm 0.07$ \\
\hline & Mianwali & $51.84 \pm 0.37^{d}$ & $7.67 \pm 0.03$ & $12.58 \pm 0.36$ & $13.95 \pm 0.21$ & $16.90 \pm 0.14^{\mathrm{abcd}}$ & $5.58 \pm 0.36$ & $6.47 \pm 0.08$ \\
\hline & Mushki & $52.60 \pm 0.73^{\mathrm{cd}}$ & $7.60 \pm 0.06$ & $14.13 \pm 0.36$ & $14.62 \pm 0.43$ & $15.81 \pm 0.17^{e}$ & $5.25 \pm 0.14$ & $6.47 \pm 0.04$ \\
\hline & Peshawari & $53.40 \pm 0.40^{c}$ & $7.66 \pm 0.04$ & $13.09 \pm 049$ & $14.04 \pm 0.42$ & $17.60 \pm 0.35^{\mathrm{ab}}$ & $5.58 \pm 0.08$ & $6.49 \pm 0.04$ \\
\hline \multirow{4}{*}{$\begin{array}{l}1.4-1.2 \\
(\mathrm{~L} 2)\end{array}$} & Lakha & $52.74 \pm 0.36^{c d}$ & $7.67 \pm 0.07$ & $13.48 \pm 0.45$ & $15.11 \pm 0.07$ & $16.21 \pm 0.15^{\text {de }}$ & $5.67 \pm 0.22$ & $6.42 \pm 0.02$ \\
\hline & Mianwali & $51.90 \pm 0.09^{d}$ & $7.63 \pm 0.08$ & $13.91 \pm 0.18$ & $13.51 \pm 0.67$ & $16.68 \pm 0.50^{\text {bcd }}$ & $5.50 \pm 0.14$ & $6.38 \pm 0.05$ \\
\hline & Mushki & $51.82 \pm 0.28^{d}$ & $7.58 \pm 0.07$ & $13.58 \pm 0.49$ & $14.35 \pm 0.35$ & $17.32 \pm 0.20^{\mathrm{abc}}$ & $5.58 \pm 0.30$ & $6.49 \pm 0.02$ \\
\hline & Peshawari & $54.70 \pm 0.33^{\mathrm{ab}}$ & $7.54 \pm 0.03$ & $13.98 \pm 1.33$ & $14.86 \pm 0.49$ & $17.69 \pm 0.18^{a}$ & $5.33 \pm 0.08$ & $6.42 \pm 0.07$ \\
\hline \multirow{4}{*}{$\begin{array}{l}1.5-1.3-1.1 \\
(\mathrm{~L} 3)\end{array}$} & Lakha & $51.98 \pm 0.53^{d}$ & $7.70 \pm 0.07$ & $13.96 \pm 0.14$ & $14.59 \pm 0.29$ & $17.15 \pm 0.20^{\mathrm{abc}}$ & $5.42 \pm 0.42$ & $6.47 \pm 0.03$ \\
\hline & Mianwali & $53.67 \pm 0.18^{\mathrm{bc}}$ & $7.68 \pm 0.09$ & $13.51 \pm 1.03$ & $14.54 \pm 0.24$ & $17.15 \pm 0.39 \mathrm{abc}$ & $5.58 \pm 0.22$ & $6.34 \pm 0.03$ \\
\hline & Mushki & $51.82 \pm 0.35^{d}$ & $7.69 \pm 0.11$ & $14.06 \pm 0.55$ & $14.37 \pm 0.45$ & $16.80 \pm 0.25^{\mathrm{bcd}}$ & $5.17 \pm 0.44$ & $6.51 \pm 0.04$ \\
\hline & Peshawari & $54.88 \pm 0.36^{a}$ & $7.56 \pm 0.02$ & $14.74 \pm 0.35$ & $14.15 \pm 0.12$ & $17.76 \pm 0.15^{\mathrm{a}}$ & $5.58 \pm 0.08$ & $6.53 \pm 0.07$ \\
\hline \multicolumn{2}{|c|}{ Source of Variation } & & & & $P$-value & & & \\
\hline \multicolumn{2}{|c|}{ LR } & 0.3169 & 0.5220 & 0.4726 & 0.5476 & 0.0177 & 0.7219 & 0.4559 \\
\hline \multicolumn{2}{|l|}{ AV } & $<.0001$ & 0.3577 & 0.6811 & 0.2520 & $<.0001$ & 0.7018 & 0.1330 \\
\hline \multicolumn{2}{|l|}{$\mathrm{LR} \times \mathrm{AV}$} & 0.0029 & 0.8580 & 0.8718 & 0.1993 & 0.0274 & 0.6527 & 0.5942 \\
\hline
\end{tabular}

Values and standard units of all parameters have been mentioned as $\mathrm{M} \pm \mathrm{SE}$, superscripted alphabets represent significant $(p \leq 0.05)$ variations, wherein $a>b>c$. .

affected by lysine and total sulfur amino acid (TSAA), while dry weight decreased when concentrations of lysine and TSAA were increased. According to them the decreased shell quality was associated with the increased synthesis of shell membranes rather than shell with less shell availability per unit area. However, another study proved that shell weight and percentage would not be affected when TSAA level was increased from 624 to $822 \mathrm{mg} / \mathrm{hen} /$ day (Shafer et al., 1996). The same effect of increasing TSAA on shell weight was also observed by Scheideler \& Elliot (1998). Albumen weight $(25.03 \pm 0.37 \mathrm{~g})$, height $(7.53 \pm 0.12 \mathrm{~mm})$, diameter $(53.09 \pm 0.41 \mathrm{~mm})$ and index $(14.19 \pm 0.15 \%)$ of birds reared on L3 was also significantly higher than those of L2 and L1 (Table 2 and 3). Albumen quality is inter-dependent upon many factors where temperature and storage time are the most crucial one (Samli et al., 2005). Significantly improved albumen weight was also reported by Garcia et al. (2005) in their study on Japanese quails fed with different levels of protein and lysine. As albumen height is the measure of egg quality and according to Khajali et al. (2008) the albumen height was not significantly $(p \leq 0.05)$ influenced when Hy-Line W36 hens were fed with reduced CP at constant TSAA to lysine ratio. Albumen height was found to decrease when kept stored for longer periods of time (Jones \& Musgroove, 2005). Significantly higher albumen height in $\mathrm{L} 3$ regimen is an indicator of good quality protein which was supplied through diet, while decrease in albumen height occurred as the eggs were aged (Silversides \& Scott, 2001). As albumen index is a measure of the quality or freshness of an egg, and like our study, significant $(p \leq 0.05)$ outcomes of albumen height, diameter, and index were reported by Muhammad et al. (2016) when Japanese quails were fed with 15, 20 and $25 \%$ CP levels. Similarly, significant variations were depicted in birds of L2 and L3 for yolk weight which was $14.46 \pm 0.27$ and $14.41 \pm 0.14 \mathrm{~g}$ (both L2 and L3 were statistically non-significant), while yolk height was higher $(p \leq 0.05)$ in L3 than L2 and L1 lysine regimens. The varieties among yolk weight $(14.61 \pm 0.18 \mathrm{~g})$ and height $(17.68 \pm 0.12 \mathrm{~mm})$ was shown by Lakha and Peshawari, respectively (Table 3). Contrarily, non-significant impact of various lysine levels was observed in Japanese quails with respect to yolk weight, height, color and index by Muhammad et al. (2016), while significant influence was reported by Garcia et al. (2005) on yolk percentage and even 
Batool T, Farooq S, Roohi N, Mahmud A, Ghayas A, Ahmad S, Usman M
Subsequent Effect of Lysine Regimens on Egg Characteristics of Native Aseel Chicken increase in yolk percentage with decreased level of protein was reported by Novak et al. (2008).

However, contrary to our findings significant influence of protein levels with respect to yolk color was described by Gunawardana et al. (2008). Significant $(p \leq 0.05)$ variations were also found in Haugh unit score among lysine regimens and Aseel varieties, among lysine regimens wherein highest score value of Haugh unit, $103.79 \pm 0.48$ was found in birds reared on $L 3$ followed by L2 $(102.09 \pm 0.87)$ and L1 (99.76 \pm 0.53$)$. Among varieties of Aseel, Peshawari showed the highest Haugh unit score than other varieties (Table 4). Haugh unit score is a measure of egg protein quality based on the height of its egg white (albumen). This test was introduced by Raymond Haugh in 1937 and is considered as an important industry measure of egg quality next to other measures such as shell thickness and strength (Chang \& Chen, 2000). Sá et al. (2007) reported that there is no influence of varying lysine (digestible) concentrations on Haugh units. Non-significant differences were observed in lysine regimens and Aseel varieties with respect to albumen and yolk $\mathrm{pH}$, yolk color, diameter as well as percent shell, albumen and yolk weight (Table 4). Gunawardana et al. (2008) demonstrated a positive impact of various dietary lysine regimens on egg composition, solids and quality of commercial brown pullets and these results were contradictory with those of Karunajeewa et al. (1987). Jardim-Filho et al. (2010) suggested a level of $600 \mathrm{mg} / \mathrm{kg}$ lysine and $15.8 \%$ CP during 24-48 weeks of age for light laying hens to produce eggs of good quality. However, a level of at least $759 \mathrm{mg} / \mathrm{hen} /$ day on an average of digestible lysine or $14 \mathrm{mg}$ lysine/egg gram was recommended by Rocha et al. (2009) for light laying hens during 24-40 weeks of age. According to Ding et al. (2016), the change in protein source and quantity had potential effect on albumen, yolk weight and color.

The yolk percentage is a linear measure of dietary $\mathrm{CP}$ at each phase indicating the interrelated association with albumen synthesis at lower levels of CP. The yolk synthesis occurs in liver and is stored in ovum till ovulation and is least affected by decreasing dietary CP levels (Novak et al., 2006). Our findings of Haugh units are in close agreement with those of Shim et al. (2013) where they also reported the significant $(p \leq 0.05)$ impact of balanced dietary protein levels on Haugh units in commercial layers during 47-54 and $63-70^{\text {th }}$ week of age.

Table 4 - Impact of various lysine regimens, Aseel varieties and their interactions on egg quality traits in native Aseel pullets.

\begin{tabular}{|c|c|c|c|c|c|c|c|}
\hline \multirow{2}{*}{\multicolumn{2}{|c|}{$\begin{array}{l}\text { Variables } \\
\text { Lysine Levels (\%)/Regimens (LR) }\end{array}$}} & Yolk diameter (mm) & Yolk index (\%) & Haugh unit score & Shell (\%) & Albumen (\%) & Yolk (\%) \\
\hline & & & & & & & \\
\hline \multicolumn{2}{|c|}{$1.3(\mathrm{~L} 1)$} & $39.52 \pm 0.18$ & $42.24 \pm 0.67^{b}$ & $99.76 \pm 0.53$ & $12.62 \pm 0.14$ & $54.54 \pm 0.35$ & $32.01 \pm 0.29$ \\
\hline \multicolumn{2}{|l|}{$1.4-1.2(L 2)$} & $39.26 \pm 0.13$ & $43.27 \pm 0.53^{\mathrm{ab}}$ & $102.09 \pm 0.87$ & $12.58 \pm 0.21$ & $54.81 \pm 0.58$ & $32.00 \pm 0.53$ \\
\hline \multicolumn{2}{|l|}{$1.5-1.3-1.1(\mathrm{~L} 3)$} & $39.47 \pm 0.33$ & $43.73 \pm 0.51^{\mathrm{a}}$ & $103.79 \pm 0.48$ & $12.83 \pm 0.16$ & $54.76 \pm 0.47$ & $31.79 \pm 0.44$ \\
\hline \multicolumn{8}{|c|}{ Aseel Varieties (AV) } \\
\hline \multicolumn{2}{|c|}{ Lakha } & $39.25 \pm 0.20$ & $42.27 \pm 0.49^{b}$ & $100.68 \pm 0.74$ & $12.31 \pm 0.12^{c}$ & $54.37 \pm 0.34$ & $32.80 \pm 0.32^{\mathrm{a}}$ \\
\hline \multicolumn{2}{|l|}{ Mianwali } & $39.31 \pm 0.17$ & $43.06 \pm 0.59^{a b}$ & $101.5 \pm 0.74$ & $12.85 \pm 0.25^{\mathrm{ab}}$ & $55.47 \pm 0.63$ & $31.08 \pm 0.53^{b}$ \\
\hline \multicolumn{2}{|l|}{ Mushki } & $39.32 \pm 0.40$ & $42.50 \pm 0.96^{b}$ & $101.69 \pm 1.07$ & $12.49 \pm 0.17^{b c}$ & $54.08 \pm 0.55$ & $32.59 \pm 0.41^{a}$ \\
\hline \multicolumn{2}{|l|}{ Peshawari } & $39.78 \pm 0.20$ & $44.48 \pm 0.33^{\mathrm{a}}$ & $103.65 \pm 0.90$ & $13.08 \pm 0.13^{\mathrm{a}}$ & $54.89 \pm 0.53$ & $31.27 \pm 0.42^{b}$ \\
\hline \multicolumn{8}{|c|}{ Lysine Levels $(\%) /$ Regimens $\times$ Aseel Varieties $(\mathrm{LR} \times \mathrm{AV})$} \\
\hline \multirow{4}{*}{$1.3(\mathrm{~L} 1)$} & Lakha & $39.54 \pm 0.12$ & $41.35 \pm 0.46^{b c}$ & $98.88 \pm 0.80$ & $12.18 \pm 0.13$ & $55.12 \pm 0.57$ & $32.18 \pm 0.73$ \\
\hline & Mianwali & $39.05 \pm 0.36$ & $43.34 \pm 0.77^{\mathrm{ab}}$ & $100.05 \pm 0.67$ & $12.71 \pm 0.13$ & $54.62 \pm 0.46$ & $31.89 \pm 0.45$ \\
\hline & Mushki & $40.07 \pm 0.52$ & $39.61 \pm 0.95^{c}$ & $103.10 \pm 0.90$ & $12.54 \pm 0.11$ & $54.03 \pm 0.58$ & $32.44 \pm 0.47$ \\
\hline & Peshawari & $39.42 \pm 0.27$ & $44.64 \pm 0.82^{a}$ & $99.33 \pm 0.92$ & $13.14 \pm 0.35$ & $54.40 \pm 0.35$ & $31.54 \pm 0.85$ \\
\hline \multirow{4}{*}{ 1.4-1.2 (L2) } & Lakha & $38.98 \pm 0.16$ & $41.63 \pm 0.55^{\mathrm{abc}}$ & $101.74 \pm 1.11$ & $12.14 \pm 0.21$ & $53.77 \pm 0.58$ & $33.61 \pm 0.16$ \\
\hline & Mianwali & $39.18 \pm 0.24$ & $42.59 \pm 1.48^{\mathrm{abc}}$ & $103.43 \pm 0.50$ & $12.88 \pm 0.67$ & $56.85 \pm 1.46$ & $29.89 \pm 1.25$ \\
\hline & Mushki & $39.36 \pm 0.29$ & $44.06 \pm 0.51^{\mathrm{ab}}$ & $99.84 \pm 1.44$ & $12.35 \pm 0.47$ & $54.28 \pm 1.08$ & $32.56 \pm 0.44$ \\
\hline & Peshawari & $39.50 \pm 0.36$ & $44.78 \pm 0.59^{a}$ & $102.52 \pm 2.97$ & $12.95 \pm 0.17$ & $54.36 \pm 0.91$ & $31.93 \pm 0.80$ \\
\hline \multirow{4}{*}{ 1.5-1.3-1.1 (L3) } & Lakha & $39.24 \pm 0.59$ & $43.82 \pm 0.72^{\mathrm{ab}}$ & $102.70 \pm 0.92$ & $12.62 \pm 0.20$ & $54.23 \pm 0.47$ & $32.61 \pm 0.38$ \\
\hline & Mianwali & $39.71 \pm 0.20$ & $43.26 \pm 1.12^{\mathrm{ab}}$ & $100.98 \pm 1.13$ & $12.97 \pm 0.53$ & $54.95 \pm 1.00$ & $31.46 \pm 0.75$ \\
\hline & Mushki & $38.53 \pm 1.01$ & $43.83 \pm 1.90^{\mathrm{ab}}$ & $104.06 \pm 1.34$ & $12.57 \pm 0.30$ & $53.93 \pm 1.46$ & $32.77 \pm 1.25$ \\
\hline & Peshawari & $40.40 \pm 0.05$ & $44.01 \pm 0.37^{a b}$ & $105.92 \pm 0.69$ & $13.16 \pm 0.21$ & $55.92 \pm 0.50$ & $30.33 \pm 0.19$ \\
\hline \multicolumn{2}{|c|}{ Source of Variation } & \multicolumn{6}{|c|}{$P$-value } \\
\hline \multicolumn{2}{|c|}{ LR } & 0.6559 & 0.0997 & 0.3210 & 0.5621 & 0.9111 & 0.8916 \\
\hline \multicolumn{2}{|l|}{ AV } & 0.4226 & 0.0409 & 0.4553 & 0.0419 & 0.3005 & 0.0130 \\
\hline \multicolumn{2}{|l|}{$\mathrm{LR} \times \mathrm{AV}$} & 0.1272 & 0.0267 & 0.8674 & 0.9896 & 0.4397 & 0.2385 \\
\hline
\end{tabular}

Values and standard units of all parameters have been mentioned as $\mathrm{M} \pm \mathrm{SE}$, superscripted alphabets represent significant $(p \leq 0.05)$ variations, wherein a $>b>c . .$. 
Batool T, Farooq S, Roohi N, Mahmud A, Ghayas A, Ahmad S, Usman M
Subsequent Effect of Lysine Regimens on Egg

Characteristics of Native Aseel Chicken

\section{CONCLUSION}

Based upon the findings of the current study, it can be concluded that provision of 1.5, 1.3, 1.1\% dietary lysine during early ages of life (1-2, 3-4 and 5-6 weeks, respectively) in Aseel birds has a positive effect on their subsequent egg traits. Additionally, the above mentioned lysine regimen (L3) interacted well with the Peshawari variety showing more pronounced improvement in egg quality. However, to achieve an improvement in persisting performance of Aseel, a detailed research work is still required.

\section{ACKNOWLEDGEMENTS}

Prof. Dr. Muhammad Akram (Late), Ex-Dean, Faculty of Animal Production and Technology, UVAS, Lahore, is greatly acknowledged for his marvelous contribution during the planning of the present research project and also the cooperation extended by UVAS administration to provide the research facilities at Indigenous Chicken Genetic Resource Centre, Department of Poultry Production, Ravi Campus, Pattoki.

\section{REFERENCES}

Adeogun IO, Amole FO. Some quality parameters of exotic chicken eggs under different storage conditions. Bulletin of Animal Health and Production in Africa 2004;52:43-47.

Ahsan-ul-Haq, Akhtar M. Poultry farming. Islamabad: Higher Education Commission; 2002. p.323-324.

Alzenbarakji N. Influence of age and strain on reproductive performance of the broiler breeder female [thesis]. Nottingham (UK): University of Nottingham; 2011.

Bain MM. Recent advances in the assessment of egg shell quality and their future application. World's Poultry Science Journal 2005;61:268-277.

Batool T, Roohi N, Mahmud A. Subsequent effects of various dietary lysine regimens on production performance, egg persistency and economics of indigenous Aseel varieties. The Journal of Animal Plant Sciences 2017;27:1-12.

Batool T. Effect of different lysine regimens on growth, productive and reproductive performance of indigenous Aseel varieties [dissertation]. Lahore (PAK): University of the Punjab; 2017.

Chang Y, Chen TC. Functional and gel characteristics of liquid whole egg as affected by $\mathrm{pH}$ alteration. Journal of Food Engineering 2000;45:237241.

Ding Y, Bu X, Zhang N, Li L, Zou X. Effects of metabolizable energy and crude protein levels on laying performance, egg quality and serum biochemical indices of Fengda-1 layers. Animal Nutrition 2016;2:9398.

Duncan DB. Multiple range and multiple F-tests. Biometrics 1955;11:1-42.

Etches RJ, Petitte JN. Reptilian and avian follicular hierarchies - models for the study of ovarian development. Journal of Experimental Zoology $1990 ; 256(S 4): 112-122$.
Garcia EA, Mendes AA, Pizzolante CC, Moreira J, Mori C, Pavan AC. Protein mehionine + cysteine and lysine levels for Japanese quails during production phase. Brazilian Journal of Poultry Science 2005;7:11-18.

Gilbert AB. Innervation of ovary of domestic hen. Quarterly Journal of Experimental Physiology and Cognate Medical Sciences 1969;54:404411.

Gunawardana P, Roland Sr DA, Bryant MM. Effect of energy and protein on performance, egg components, egg solids, egg quality, and profits in molted Hy-Line W-36 hens. Journal of Applied Poultry Research 2008;17:432-439.

Holsheimer JP, Ruesink EW. Effect on performance, carcass composition, yield, and financial return of dietary energy and lysine levels in starter and finisher diets fed to broilers. Poultry Science 1993;72(5):806-815.

Hussain S, Ahmed Z, Khan MN, Khan TA. A study on quality traits of chicken eggs collected from different areas of Karachi. Sarhad Journal of Agriculture 2013;29:255-259.

Jardim-Filho RM, Stringhni JH, Andrade MA, Café MB, Leandro NSM, Carvalho FB. Levels of digestible lysine for Hy-Line W-36 hens in production period. Brazilian Journal of Animal Science 2010;39:787795.

Jones DR, Musgrove MT. Effects of extended storage on egg quality factors. Poultry Science 2005;84:1774-1777.

Karunajeewa H, Abu-Serewa S, Tham SH, Eason P. The effects of dietary level of sunflower seeds and lysine on egg quality and laying performance of White Leghorn hens. Journal of the Science of Food and Agriculture 1987;41:325-333

Khajali F, Khoshouie EA, Dehkordi SK, Hematian M. Production performance and egg quality of Hy-line w36 laying hens fed reduced-protein diets at a constant total sulfur amino acid: lysine ratio. Journal of Applied Poultry Research 2008;17:390-397.

Kidd MT, Kerr BJ, Halpin KM, McWard GW, Quarles CL. Lysine levels in starter and grower-finisher diets affect broiler performance and carcass traits. Journal of Applied Poultry Research 1998;7(4):351-358.

Kul S, Seker I. Phenotypic correlations between some external and internal egg quality traits in the Japanese quail. International Journal of Poultry Science 2004;36:400-405.

Leeson S, Zubair AK. Nutrition of the broiler chicken around the period of compensatory growth. Poultry Science 1997;78:992-999.

Leeson S, Summers JD. Commercial poultry nutrition. $3^{\text {rd }}$ ed. Nottingham: Nottingham University Press; 2005.

Muhammad N, Altine S, Abubakar A, Chafe UM, Saulawa LA, Garba MG, et al. Effect of varying protein levels and preservation methods on egg production performance and internal egg qualities of Japanese quails in a semi-arid environment. European Journal of Basic and Applied Science 2016;3:8-19.

Murphy ME. Amino acid composition of avian eggs and tissues: Nutritional implications. Journal of Avian Biology 1994;25:27-38.

Novak CL, Yakout H, Scheideler SE. The combined effects of dietary lysine and total sulfur amino acid level on egg production parameters and egg components in Dekalb Delta laying hens. Poultry Science 2004;83:977-984.

Novak $\mathrm{CL}$, Yakout $\mathrm{H}$, Scheideler SE. The effect of dietary protein level and total sulfur amino acid: Lysine ration on egg production parameters and egg yield in Hy-Line W-98 hens. Poultry Science 2006;85:21952206 . 
Batool T, Farooq S, Roohi N,

Mahmud A, Ghayas A, Ahmad S, Usman M

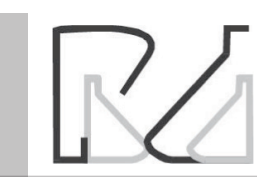

Subsequent Effect of Lysine Regimens on Egg Characteristics of Native Aseel Chicken
Novak CL, Yakout HM, Remus J. Response to varying dietary energy and protein with or without enzyme supplementation on leghorn performance and economics. 2. Laying period. Journal of Applied Poultry Research 2008;17:17-33.

NRC - National Research Council. Nutrient requirements of poultry. $9^{\text {th }}$ ed. Washington: National Academic Press; 1994.

Onagbesan O, Bruggeman V, Desmit L, Debonnr M, Witters A, Tona K, Everaert N, Decuypere E. Gas exchange during storage incubation of avian eggs; effects on embryogenesis, hatchability, chick quality and post hatch growth. World's Poultry Science Journal 2007;63:557-573.

Rocha TC, Gomes PC, Donzele JL, Barreto SLT, Mello HHC, Brumano G. Digestible lysine levels in feed for 24 to 40-week old laying hens. Brazilian Journal of Animal Science 2009;38:1726-1731.

Sá LM, Gomes PCG, Rostagno HS, Albino LFT, Agostini P. Nutritional requirement of lysine for laying hens in the period from 34 to 50 weeks old. Brazilian Journal of Animal Science 2007;36:1829-1836.

Samli HE, Agma A, Senkoylu N. Effect of storage time and temperature on egg quality in old laying hens. Journal of Applied Poultry Research 2005; 14:548-553.

Scheideler SE, Elliot MA. Total sulfur amino acid (TSAA) intake to maximize egg mass and feed efficiency in young layers (19-45 week of age). Poultry Science 1998;77(1 Suppl):130.

Seidler E. FAO, egg marketing. A guide for the production and sale of eggs [Agriculture Services Bulletin 150]. Rome; 2003.

Shafer DJ, Carey JB, Prochaska JF. Effect of dietary methionine intake on egg component yield and composition. Poultry Science 1996;75:10801085.
Shim MY, Song E, Billard L, Aggrey SE, Pesti GM, Sodsee P. Effects of balanced dietary protein levels on egg production and egg quality parameters of individual commercial layers. Poultry Science 2013;92:2687-2696.

Sibbald IR, Wolynetz MS. Effects of dietary lysine and feed-intake on energy-utilization and tissue synthesis by broiler chicks. Poultry Science 1986;65(1):98-105

Silversides FG, Korver DR, Budgell KL. Effect of strain of layer and age at photo stimulation on egg production, egg quality and bone strength. Poultry Science 2006;85:1136-1144.

Silversides FG, Scott TA. Effect of storage and layer age on quality of eggs from two lines of hens. Poultry Science 2001;80:1240-1245.

Steel RGD, Torri JH, Dicky DA. Principles and procedures of statistics, a biochemical approach. $3^{\text {rd }}$ ed. New York: McGraw Hill Book; 1997.

Tumova E, Gous RM. Interaction of hen production type, age and temperature on laying pattern and egg quality. Poultry Science 2012;91:1269-1275.

Usman M, Basheer A, Akram M, Zahoor I. A comparative study of production performance and egg quality parameters of Naked-Neck and indigenous Aseel chicken of Pakistan. Journal of Basic \& Applied Sciences 2014;10:160-163.

Valério SR, Oliveira RF, Donzele JL, Gomes PC, Apolônio LR, Resende WO. Levels of digestible lysine in diets, where the amino acid ratio was maintained, was maintained for 22 to 42-day-old broilers kept under heat stress. Revista Brasileira de Zootecnia. 2003; 32(2): 372-82. 